\title{
On properties of countable character
}

\section{Gabriel Sabbagh}

\begin{abstract}
It is proved that if a class $X$ of algebras of countable similarity type is closed under isomorphism and ultrapower, then the class of subalgebras of direct products of elements of $X$ is of countable character.
\end{abstract}

\section{Introduction}

This short paper is composed of variations on a theme of B.H. Neumann. In a recent talk in Nice, he introduced the notion of property of countable character and showed that several properties are of countable character. Various persons, including W.W. Boone, A. Robinson and the author, suggested the possibility of using a kind of Löwenheim-Skolem Theorem for deriving such results. Although the most obvious tool seems to be the downward Löwenheim-Skolem Theorem for $L_{\omega_{1} \omega}$ (cf. [6]) and it is possible to describe in an infinitary language universal properties of countable character, the main purpose of this note is to show how to use the ordinary Löwenheim-Skolem-Tarski Theorem [13] for unifying and improving some of the results of [9].

\section{Preliminaries}

For simplicity we will only deal with algebras, namely with sets endowed with an arbitrary number of finitary operations (functions), some of which may be of arity $0 . A$ being an algebra, we denote by $\alpha_{n}(A)$ the cardinal of the set of operations of arity $n$ of the algebra $A$.

Received 12 October 1970. The author would like to thank Professor B.H. Neumann for allowing him to see a typescript of [9] before publication. 
The sequence $\left\langle\alpha_{n}(A)\right\rangle_{n \in \omega}$ is called the similarity type of $A$. We denote by $X$ a nonvoid fixed class closed under isomorphism of algebras, all of which have the same similarity type. We denote by $\alpha=\alpha(X)$ the cardinal $\sum_{n \in \omega} \alpha_{n}(A)$ where $A$ is an element of $X$. To $X$ is associated in the usual way a first-order language, the cardinal of which will be denoted by $\gamma$ and coincides with $\sup \left(\alpha, \kappa_{0}\right)$. Except if otherwise stated, all the logical concepts are considered with respect to this language.

As usual, we denote by $S X$ (respectively $P X$, respectively $R X$ ) the class of algebras isomorphic to subalgebras (respectively cartesian products, respectively subcartesian products) of elements of $X$ ([4], [8]). If $X$ coincides with $S X, X$ is said to be universal. If $X$ coincides with the class of finite algebras, $R X$ is said to be the class of residually finite algebras. In general one has

$$
\begin{aligned}
& R X \subseteq S R X=R S X=S P X \\
& P S X \subseteq S P X ; \quad S S X=S X .
\end{aligned}
$$

For every infinite cardinal $\beta$, we denote by $L_{\beta}(X)$ the class of algebras all of whose subalgebras generated by strictly less than $\beta$ elements belong to $X$. We may then introduce the following

DEFINITION. $X$ is said to be of $\beta$-character if $L_{B}(X)$ is included in $X$.

We will adapt the terminology of [9] in saying that $X$ is of local character (respectively countable character) if $X$ is of $\kappa_{0}$-character (respectively $\aleph_{1}$-character). The definitions of [9] are different from ours but coincide with them if $X$ is universal and if $\alpha$ is strictly less than $\kappa_{1}$.

\section{Main section}

We can now state our result.

THEOREM 1. Let $\delta$ be the successor cardinal of $\gamma$. If $X$ is closed under ultrapower, then SPX is of $\delta$-character. 
Proof. The proof is best summarized by the following diagram:

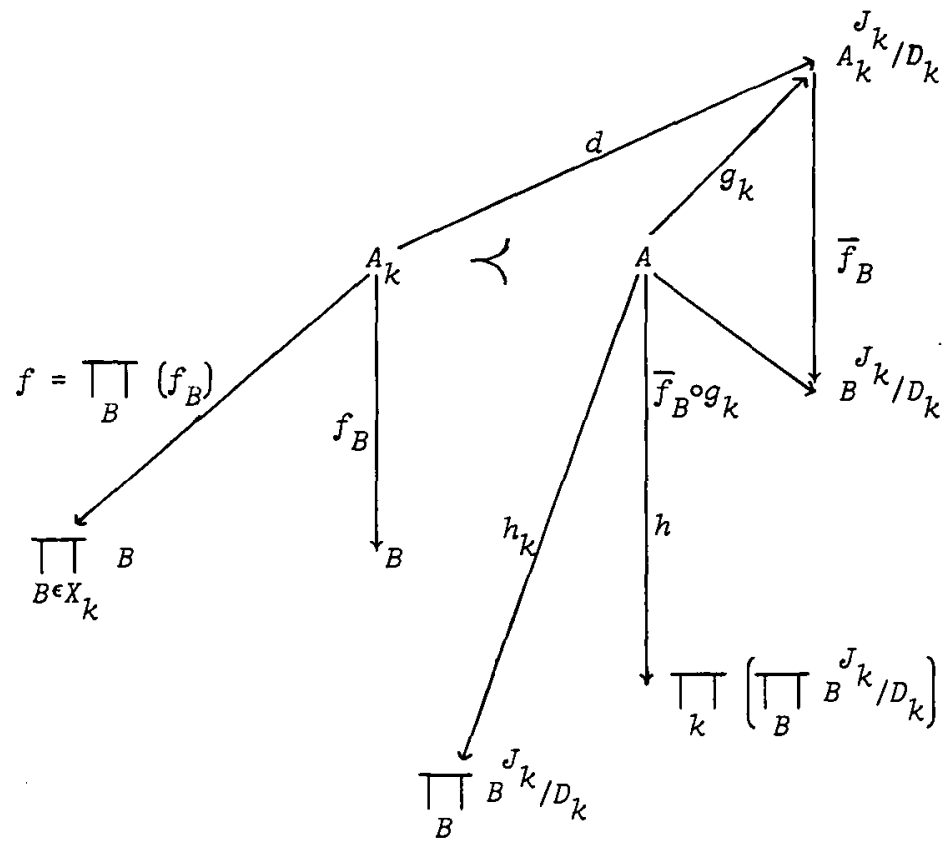

Let $A$ be an arbitrary element of $L_{\delta}(S P X)$. We wish to prove that $A$ is an element of $S P X$. We can clearly assume that $A$ is of cardinal $\geq \gamma$. By the Löwenheim-Skolem-Tarski Theorem, every subset $k$ of cardinal 2 of $A$ is contained in an elementary substructure $A_{k}$ of $A$ of cardinal $\gamma$. By assumption, there exist a family $X_{k}$ of elements of $X$ and for each element $B$ of $X_{k}$ a homomorphism $f_{B}$ of $A_{k}$ into $B$ such that the "product" homomorphism $f=\prod_{B}\left(f_{B}\right)$ of $A_{k}$ into $\prod_{B \in X_{k}} B$ is one-one. By Scott's Lemma ([2], p. 163), since $A_{k}$ is an elementary substructure of $A$, there exists a one-one homomorphism $g_{k}$ of $A$ into an ultrapower $A_{k}{ }^{j} / D_{k}$ of $A_{k}$ whose restriction to $A_{k}$ coincides with the canonical embedding $d$ of $A_{k}$ into $A^{J} k / D_{k}$. For each element $B$ of 
$x_{k}, f_{B}$ induces a homomorphism $\bar{f}_{B}$ of $A_{k}^{J} / D_{k}$ into ${ }_{B}^{J} k / D_{k}$. The family $\left(f_{B}^{\circ} \circ g_{B \in X_{k}}\right.$ allows us to define a homomorphism $h_{k}=\prod_{B \in X_{k}}\left(f_{B}^{\circ} \circ g_{k}\right)$ of $A$ into $\prod_{B \in X_{k}}\left(B^{J} k / D_{k}\right)$.

Let $K$ now denote the set of all two-element subsets $k$ of $A$. The family $\left(h_{k}\right)_{k \in K}$ allows us to define a homomorphism $h=\prod_{k}\left(h_{k}\right)$ of $A$ into $M=\prod_{k \in K}\left(\prod_{B \in X_{k}}\left(B^{J} / D_{k}\right)\right)$. Since by assumption $X$ is closed under ultrapower, $M$ is an element of $P X$. For proving that $A$ is an element of $S P X$, it now suffices to show that $h$ is one-one.

Let $a$ and $b$ be two distinct elements of $A$. Let $q$ denote the subset $\{a, b\}$ of $A$. There exists an element $B$ of $X_{q}$ such that $f_{B}(a) \neq f_{B}(b)$. It easily follows that $\bar{f}_{B}(d(a))$ and $\bar{f}_{B}(d(b))$ are distinct and hence that $\bar{f}_{B} \circ g_{q}(a)$ and $\bar{f}_{B} \circ g_{q}(b)$ are distinct. We then obtain $h_{q}(a) \neq h_{q}(b)$, which implies $h(a) \neq h(b)$. The proof is finished.

COROLLARY 1. Let $\delta$ be the successor cardinal of $\gamma$. If $X$ is closed under ultrapower and is universal, then $R X$ is of $\delta$-character.

Proof. Since $X$ is universal, (1) implies that $R X$ is equal to $S P X$. One then applies the theorem.

Corollary 1 yields under weaker assumptions Theorem 3 and Theorem 4 of [9]. Theorem 3 essentially states that if $X$ is the union of a family of quasivarieties, then $R X$ is of $\delta$-character. A quasivariety is just a universal Horn class of algebras ([4], p. 235). It is now plain that Theorem 3 remains true if one only assumes that $X$ is the union of a family of universal elementary (in the wider sense) classes of algebras. It is perhaps worthwhile to state formally our version of Theorem 4; its only advantage is that $\alpha$ need not be finite. 
COROLLARY 2. If $\alpha$ is countable, the class of residually finite algebras is of countable character.

Proof. Indeed, an ultrapower of a finite set is finite.

\section{Other approaches}

1. The obvious strengthening of Corollary 2 and of Theorem 1 fails: the class of residually finite commutative groups is not of local character; indeed every finitely generated commutative group is residually finite, while a non-trivial divisible group is never residually finite. However, if one assumes in the theorem that $X$ is closed under ultraproduct, one may conclude that SPX is of local character. For proving that fact, it is enough by a standard embedding theorem (see for example [3] which has a nearly complete bibliography, [7] or [10]) to establish the following

LEMIA. If $X$ is closed under ultraproduct, then SPX is a universal elementary class.

Proof. The shortest way is to derive the lemma from a similar, slightly weaker, result of Vaught [14]. According to that result, if $Y$ is an elementary class (or even a $P C_{\triangle}$ class), then $S P Y$ is a universal elementary class. Let us denote by $X^{\prime}$ the elementary class generated by $X$, namely the class of models of all sentences valid in all elements of $X$. It is easy to see that $X^{\prime}$ is the class of the algebras which are elementarily embeddable in an element of $X$. (A more general result is given in [11].) One then has $S P X \subseteq S P X^{\prime} \subseteq S P S X$; by (2) one obtains $S P S X \subseteq S P X$ and hence one has $S P X=S P X^{\prime}$. Since $X^{\prime}$ is an elementary class, the proof is finished.

As an immediate application, one has

COROLLARY 3. Let $n$ be a positive integer and let $X_{n}$ be the class of (finite) algebras of cardinal $<n . R X_{n}$ is of local character.

Corollary 3 is implicit in [9].

2. As mentioned in the introduction and expounded in [6], it is tempting to try to use some infinitary logic for proving that a given 
class is of countable character. In some cases, it is enough to consider the language $L_{\omega_{1} \omega}$ : for example, let $N$ be the class of nilpotent groups. It is easy to find a sentence $\sigma$ of the $L_{\omega_{1} \omega}$ theory of groups such that $N$ is the class of models of $\sigma$. If $N$ were not of countable character, there would exist a group $G$ such that $G$ is a model of 70 and every countable subgroup of $G$ is a model of $\sigma$, which would contradict the Löwenheim-Skolem theorem for $L_{\omega_{1} \omega}$.

On the other hand, as noticed in conversation with A. Macintyre, there are many classes which are of countable character and which are not definable in $L_{\omega_{1} \omega}$ (nor in $L_{\infty \omega}$ ). Two simple examples are the class of commutative reduced $p$-groups ([1], Theorem 2.4) and the class of noetherian rings ([5], Theorem 11). It is not hard in fact to give a syntactical characterization of universal classes of countable character if one is willing to devise an ad hoc language:

THEOREM 2. Let $B$ be an infinite cardinal. Let $\mu$ denote the cardinal $\sup (B, \gamma)$. If $X$ is universal, then the following assertions are equivalent:

(i) $X$ is of $\beta$-character;

(ii) there exists a set $S$ of sentences $\dot{\psi}$ of the form

$$
\psi=\left(\forall x_{1}\right) \ldots\left(\forall x_{\lambda}\right) \ldots \lambda_{\lambda<\rho<\beta} \varphi\left(x_{\lambda}\right)
$$

where $\rho$ is a cardinal $<\beta(\rho$ depends upon $\psi)$ and $\varphi\left(x_{\lambda}\right)$ is a quantifier-free formula of $L_{\infty \infty \infty}$, that is a quantifier-free formula of possibly infinite length, such that $X$ is the class of models of $S$;

(iii) there exists a set $T$ of sentences $\psi$ of the form

$$
\psi=\left(\forall x_{1}\right) \ldots\left(\forall x_{\lambda}\right) \ldots \lambda_{\lambda<\rho<\beta} \varphi\left(x_{\lambda}\right)
$$

where $\rho$ is a cardinal $<\beta$ and $\varphi\left(x_{\lambda}\right)$ is a disjunction of length at most equal to $\mu$ of atomic formulas and negations of atomic formulas, such that $X$ is the class of models of $T$. 
Proof. $(i i i)=(i i)$ is obvious and $(i i) \Rightarrow(i)$ is easy. For establishing the implication $(i) \Rightarrow$ (iii) we will follow an argument due to Tarski ([12], Theorems 1.1 and 1.2 ).

We will first prove a more precise version of a consequence of this implication. Let $A$ be an algebra of the same similarity type as $X$ and let $Y$ be the class of algebras of the same similarity type as $X$ into which $A$ cannot be embedded. We assume that $A$ admits a generating subset of cardinal $\rho<\beta$. We want to show that there exists a single sentence $\psi_{A}$ of the form described in (iii) such that $Y$ is the class of models of $\psi_{A}$.

Let $\left(a_{\lambda}\right)_{\lambda<\rho}$ be a non-repeating enumeration of a generating subset of $A$. Let $F$ be the algebra of words of the same similarity type as $X$ freely generated by a set $\left\{x_{\lambda}\right\}_{\lambda<p}$ of distinct elements. To each pair $C=\left\{P\left(x_{\lambda}\right), Q\left(x_{\lambda}\right)\right\}$ of words of $F$ we associate the formula $U_{C}$ defined as follows:

$U_{C}= \begin{cases}P\left(x_{\lambda}\right)=Q\left(x_{\lambda}\right) & \text { if the elements } P\left(a_{\lambda}\right) \text { and } Q\left(a_{\lambda}\right) \text { of } A \text { are } \\ & \text { distinct, } \\ P\left(x_{\lambda}\right) \neq Q\left(x_{\lambda}\right) & \text { if the elements } P\left(a_{\lambda}\right) \text { and } Q\left(a_{\lambda}\right) \text { of } A \text { are equal. }\end{cases}$ It is easy to check that one can take for $\psi_{A}$ the formula

$$
\left(\forall x_{1}\right) \cdots\left(\forall x_{\lambda}\right) \cdots \operatorname{lip}_{C}\left(\bigvee_{C} U_{C}\right)
$$

We now proceed to the proof of the general case. Let $T$ be the set of all sentences of the form given in (iii) which are valid in all the elements of $X$. Assuming that $X$ is universal and of B-character, we will show that $X$ is the class of models of $T$. It clearly suffices to prove that an arbitrary model $M$ of $T$ is an element of $L_{\beta}(X)$. Let $B$ be a subalgebra of $M$ generated by strictly less than $\beta$ elements. It is plain that the sentence $\psi_{B}$ is not an element of $T$. It follows that $B$ can be embedded in an element of $X$. Since $X$ is universal, the 
proof is complete.

It is easy to derive from the previous theorem a straightforward generalization of Theorem 2 of [9].

COROLLARY 4. Let $B$ be an infinite cardinal and let $I$ be a set of cardinal strictly less than the smallest cardinal co-final with $\beta$. The union of a family indexed by $I$ of universal classes of $\beta$-character is a universal class of $\beta$-character.

We have been unable to deduce Theorem 1 from Theorem 2. A more interesting question would be to know if there exists a $L_{\omega_{l} \omega}$ analogue of the result of Vaught previously mentioned. We do not even know if the class of residually finite groups is definable in $L_{\omega_{l} \omega}$; of course, the class of commutative residually finite groups is.

3. Theorem 1 constitutes a model-theoretic generalization of Corollary 2. There is a different generalization, which is due to $\mathrm{J}$. Mycielski and is included here with his kind permission.

THEOREM 3. (Mycielski) Let $\delta$ be the successor cardinal of $\gamma$. If $X$ is a class of atomic compact algebras, then SPX is of $\delta$-character.

Proof. Let $A$ be an arbitrary element of $L_{\delta}(S P X)$. We wish to prove that $A$ is an element of SPX. We can clearly assume that $A$ is of cardinal $\geq \gamma$. By the Löwenheim-Skolem-Tarski Theorem, every subset $k$ of cardinal 2 of $A$ is contained in an elementary substructure $A_{k}$ of $A$ of cardinal $\gamma$. By assumption, there exists an embedding of $A_{k}$ into a product $B_{k}$ of elements of $X$. By [15], p. $107 B_{k}$ is atomic compact. By a well-known theorem of Weglorz, a version of which may be found in [15], p. 105, the embedding of $A_{k}$ into $B_{k}$ can be extended to a homomorphism $h_{k}$ of $A$ into $B_{k}$. It is easy to see that the "product" homomorphism $\prod_{k}\left(h_{k}\right)$ of $A$ into $\prod_{k} B_{k}$ is an embedaing and makes $A$ an element of $S P X$.

To derive Corollary 2 from Theorem 3, it is enough to use the fact 
that every finite algebra, and more generally every (Hausdorff) compact algebra, is atomic compact ([15], p. 75).

Note added in proof. A version of Theorem 2 appears in Tarski's paper, "Remarks on predicate logic with infinitely long expressions", Colloq. Math. 6 (1958), 171-176.

\section{References}

[1] J. Barwise and P. Eklof, "Infinitary properties of torsion abelian groups", Ann. Math. Logic (to appear).

[2] J.L. Bell and A.B. SIomson, Models and ultraproducts: an introduction (North-Holland, Amsterdam, London, 1969).

[3] John P. Cleave, "Local properties of systems", J. London Math. Soc. 44 (1969), 121-130.

[4] P.M. Cohn, Universal algebra, (Harper \& Row, New York, Evanston, London, 1965).

[5] P. Eklof and Gabriel Sabbagh, "Definability problems for modules and rings", submitted to J. Symbolic Logic.

[6] R.D. Kopperman and A.R.D. Mathias, "Some problems in group theory", The syntax and semantics of infinitary languages, 131-138 (Lecture notes in Mathematics, 72, edited by Jon Barwise; Springer-Verlag, Berlin, Heidelberg, New York, 1968).

[1] B.H. Neumann, "An embedding theorem for algebraic systems", Proc. London Math. Soc. (3) 4 (1954), 138-153.

[8] B.H. Neumann, "Special topics in algebra: Universal algebra", notes by P.M. Neumann, (Courant Institute of Mathematical Sciences, New York University, New York, 1962).

[9] B.H. Neumann, "Properties of countable character", Proc. Internat. Congr. Math. (Nice, 1970), (to appear).

[10] A. Robinson, "Note on an embedding theorem for algebraic systems", J. Londion Math. Soc. 30 (1955), 249-252. 
[11] Gabriel Sabbagh, "A note on the embedding property", submitted to Math. z.

[12] Alfred Tarski, "Contributions to the theory of models. I", Nederl. Akad. Wetensch. Proc. Ser. A. 57 (1954), 572-581.

[13] Alfred Tarski and Robert L. Vaught, "Arithmetical extensions of relational systems", Compositio Math. 13 (1957), 81-102.

[14] Robert Vaught, "The elementary character of two notions from general algebra", Essays on the foundations of mathematics, 226-233. (Magnes Press, Hebrew University, Jerusalem, 1961).

[15] G.H. Wenzel, "Compactness in algebraic structures", (Report 68-31, Carnegie-Mellon University, Pittsburg, 1968).

1, square François Couperin,

92-Antony,

France. 